\title{
Prevalence of cystic macular lesions in patients with Usher II syndrome
}

\begin{abstract}
Purpose To evaluate the prevalence of cystic macular lesions in patients with Usher II syndrome.

Methods All Usher type II patients seen in the inherited eye disease clinic at the University of Illinois at Chicago between January 2002 and December 2007 were included $(n=76)$. Each participating patient underwent a detailed clinical examination, including best-corrected visual acuity, slitlamp biomicroscopy and dilated fundus examination. The presence of cystoid lesions was determined by optical coherence tomography (OCT), fundus fluorescein angiogram (FFA), fundus photographs and/or clinical examination.

Results A cystic-appearing macular change was observed in at least one eye in 19 out of the 76 patients (25\%), 13 on the basis of OCT, five using FFA (two solely with the use of FFA and three based on clinical notes and FFA findings) and one based solely on clinical notes. Of the 18 patients with CME, determined by OCT or FFA, five $(\mathbf{2 7 . 8 \%})$ showed either a funduscopically normal-appearing macula $(n=4)$ or an atrophic appearing macular change $(n=1)$.

Conclusions One-fourth of our total cohort of Usher II patients had cystic macular lesions. Moreover, a funduscopically normal-appearing macula was observed in $22 \%(n=4)$ of our 18 patients with cystic-appearing macular lesions on OCT and/or FFA testing. On the basis of the reasonably high prevalence of cystic macular lesions in our cohort, it would seem prudent to evaluate Usher II patients for the presence of cystoid macular oedema.
\end{abstract}

Eye (2009) 23, 1206-1209; doi:10.1038/eye.2008.105; published online 18 April 2008

Keywords: Usher syndrome type II; cystoid macular oedema; optical coherence tomography; fundus fluorescein angiography
S Walia, GA Fishman and M Hajali

\section{Introduction}

Usher syndrome is an autosomal recessive group of disorders most often characterized by non-progressive sensorineural hearing loss and retinitis pigmentosa (RP). Usher syndrome type II is the most common form, ${ }^{1-4}$ with onset of visual impairment in the second decade, congenital sensorineural hearing loss, most severe at high frequencies, and absence of vestibular dysfunction. ${ }^{5,6}$

Previous studies have categorized the macular findings in Usher patients as either normal, showing cystoid macular oedema (CME), macular holes, or an atrophic-appearing lesion. ${ }^{7,8}$ The prevalence of CME has been reported to be $8 \%$ in Usher II patients on the basis of clinical findings. ${ }^{8}$

Optical coherence tomography (OCT) has proven to be one of the most sensitive modalities for diagnosing CME. ${ }^{9,10}$ Using the OCT, cystic-appearing macular changes may be detected in patients even in the absence of dye leakage on fluorescein angiography. ${ }^{11-13}$

Cystoid changes in the macula may further lead to a lamellar or full-thickness macular hole $\mathrm{e}^{14,15}$ and likely contribute to progressive impairment of visual acuity. Various options are now available for the treatment of CME in RP patients. ${ }^{16-23}$ The purpose of the present study was to determine the prevalence of $\mathrm{CME}$ in Usher II patients.

\section{Patients and methods}

All Usher II patients with various degrees of disease severity, seen at the University of Illinois at Chicago between January 2002 and December 2007 by one of the authors (GAF) were included in the study. The diagnosis of Usher II syndrome was based on a history of night blindness, presence of characteristic fundus changes including waxy disc pallor, attenuated retinal vessels and bone spicule-like \author{
work. \section{The authors do not have any
proprietary interest in this \\ Received: 25 January 2008 12 March 2008 \\ Published online: 18 April 2008}


pigmentation, reduced or non-detectable rod and cone electroretinogram amplitudes, impaired peripheral visual fields, history of moderate congenital sensorineural hearing loss and the clinical absence of vestibular disturbances. Although detailed audiometric tests were not available on all patients, there were no patients with profound congenital sensory hearing loss, unintelligible speech, or a history of vestibular disturbance who were included in the study. This study was approved by an institutional review board at the University of Illinois and was conducted in accordance with tenets of the Declaration of Helsinki. Informed consent was obtained from the study participants. The examinations were conducted in accordance with Health Insurance Portability and Accountability Act regulations.

A total of 76 patients were included. A number of patients were contacted either through phone and/or e-mail, others were enrolled at the time of their routine clinical follow-up examination $(n=8)$, while an additional number $(n=19)$ were included who were evaluated as part of another study which correlated foveal structural and functional changes with OCT determined foveal thickness measurements. The exclusion criteria included a prior history of uveitis, central retinal vein occlusion or diabetic retinopathy. Pseudophakic patients with posterior chamber intraocular lens implants, aphakic patients with an intact posterior capsule and diabetic patients without any evidence of diabetic retinopathy were not excluded from the study.

For each patient, a complete eye examination was obtained including best-corrected visual acuity using a Snellen chart, slit-lamp biomicroscopy and intraocular pressure testing using a Goldmann applanation tonometer. Both pupils were dilated with $1 \%$ Tropicamide and $2.5 \%$ Phenylephrine. The patients then underwent a detailed fundus examination with a +78 diopter lens stereobiomicroscopy, and both direct and indirect ophthalmoscopy.

OCT was performed on all patients who could be contacted and were willing to participate in OCT testing. In some patients $(n=22)$ a time domain OCT (StratusOCT, Carl Zeiss Meditec Inc., Dublin, CA; software version 4.0.1) was used, while in others $(n=16)$ we used a Fourier domain OCT unit (RTVue, Optovue Inc., Fremont, CA; software versions 1.2.6 and 2.0.3.2). Six radial lines of $6-\mathrm{mm}$ each at $30^{\circ}$ intervals passing through the center of the fovea were used for the time domain OCT testing. Each of these six lines was acquired individually and sequentially by the operator and consisted of 512 A-scans. For the Fourier domain OCT testing, the radial slicer protocol was used. It consisted of twelve $6-\mathrm{mm}$ radial scans at $15^{\circ}$ intervals passing through the center of the fovea. All the 12 scans were acquired together at the same time and the total time taken for their acquisition was $0.27 \mathrm{~s}$. Each radial line consisted of 1024 A-scans.

For patients who could either not be contacted or were unwilling to return for OCT testing, previous records were utilized including fundus fluorescein angiograms (FFA), fundus photographs and clinical notes from prior visits. A patient was considered to have CME if there was evidence of CME at any one visit to the University of Illinois. The macula in each patient was described as normal (including patients with a blunted foveal reflex or epiretinal membrane), showing cystoid macular oedema, partial- or full-thickness macular hole, or atrophic change (including patients with either a bulls-eye-like lesion or geographic-appearing lesion).

\section{Results}

Our cohort consisted of 76 patients from 71 families, with a mean age of 38 years and age range of 8-68 years. Total number of female patients was 37 (48.7\%). Depending on race, the patient group could be divided into 62 Caucasians (81.6\%), 7 African-Americans (9.9\%), 5 Hispanics (6.6\%) and 2 Asians (2.6\%).

The visual acuity of the patients ranged from $20 / 15^{-2}$ to light perception. Eighteen patients were pseudophakic with the presence of a posterior chamber intraocular lens implant in at least one eye. One additional patient was aphakic in both eyes.

Optical coherence tomography was obtained on 38 patients (time domain on 22 patients and Fourier domain on 16 patients). Thirty-three of these 38 patients had OCT alone and five had both FFA and OCT. Another eight patients had FFA alone. Of the remaining 30 patients, 16 had clinical notes and $60^{\circ}$ color fundus photographs while in 14 only clinical notes which described the macula were used.

On the basis of clinical examination, 15 patients had bilateral atrophic-appearing lesions (19.7\%), 14 had a cystic-appearing lesion in at least one eye (18.4\%) of which four patients had a cystic-appearing change in one eye only, 45 had a normal-appearing macula bilaterally $(59.2 \%)$ while two patients had a full-thickness macular hole in at least one eye (2.6\%). One of these two patients with a macular hole had this lesion in one eye and an atrophic-appearing lesion in the other.

A cystic-appearing macular change was observed at least in one eye in 19 out of 76 patients (25\%), 13 on the basis of OCT, five using FFA (two solely with the use of FFA and three based on clinical notes and FFA findings) and one based solely on clinical notes. The clinical appearance of the macula in 19 patients with CME was: nine patients had clinically-evident cysts bilaterally, four had a normal-appearing macula in both eyes (CME was 
observed in both eyes using OCT/FFA), one had an atrophic-appearing lesion bilaterally (CME was detected in one eye using OCT), one had cystic-like lesions in both eyes (a partial-thickness hole was detected in one eye and CME in the other), two had CME in one eye and a normal-appearing macula in the other and two patients had CME in one eye and a normal-appearing macula in the other (OCT scans were available only in one eye with CME in these two patients).

OCT was performed on 38 patients while an additional eight patients had FFA, for a total of 46 patients. Eighteen among these 46 patients had CME (39.1\%). Of these 18 patients with CME, determined by OCT $(n=13)$ or FFA $(n=5)$, five $(27.8 \%)$ patients had either a normalappearing macula $(n=4)$ or an atrophic-appearing lesion $(n=1)$ on fundus examination. Hence, it is likely that by clinical fundus examination alone we would have underestimated the prevalence of CME in our total cohort of 76 Usher syndrome II patients. If we consider the above figure of $27.8 \%$, it is hypothetically conceivable that at least approximately $1 / 4$ of our additional 30 patients (who did not have OCT and/or FFA) could potentially have had CME $(n=8)$, making a predictive total number with $\mathrm{CME}$ to 27 (35.5\%) in our entire cohort of 76 patients. This percentage would be similar to the $39.1 \%$ of 46 patients with CME determined by OCT, fluorescein angiography or both.

The average age of patients with CME in our cohort was 38 years, while those with atrophic-appearing lesions had an average age of 49.5 years. The mean age of patients who had OCT and/or FFA testing was 41.7 years compared to a mean age of 32.5 years for patients who did not undergo these tests.

\section{Discussion}

Previous studies have reported the presence of CME in patients with Usher syndrome and non-syndromic retinitis pigmentosa. Increased leakage of fluid from the outer and inner blood-retinal barriers has been reported to result in $\mathrm{CME} .^{24}$ Increased inward permeability and decreased outward permeability to fluorescein by vitreous fluorophotometry have been documented. ${ }^{25}$ Studies have also postulated the role of antiretinal antibodies in triggering CME in RP patients. ${ }^{26}$

The prevalence of cystic-appearing lesions in the macula in our study of 76 Usher II patients was found to be $25 \%$. Fishman et $a l^{8}$ have previously reported a figure of $8 \%$ and Tsilou et $a l^{6}$ documented the prevalence of CME to be $9 \%$, based on clinical examination. In another study by Schwartz et $a l^{27}$, six out of 10 Usher II patients were found to have CME with the use of OCT. The higher prevalence of cystic lesions in our cohort of 46 patients who were tested by OCT, FFA or both as compared to some of the previous studies may be due to the use of OCT, and particularly high resolution OCT, making it more sensitive than clinical examination alone in identifying these lesions.

The prevalence of clinically apparent cystic lesions was similar to clinically apparent atrophic lesions in our patient group, although the mean age of patients with atrophic lesions was higher (48 years vs 38 years). A previous report documented a higher prevalence of atrophic than cystic lesions in Usher II patients. ${ }^{8}$ It is conceivable that a different cohort of Usher II patients with a different age range as compared to our group may show a somewhat different percentage for the prevalence of CME.

In the present study, 19 pseudophakic or aphakic patients were not excluded from the study. The prevalence of cystic-like lesions in these pseudophakic and aphakic patients $(26.3 \%)$ was observed to be similar to either the total patient group (25\%) or to those who were phakic $(24.56 \%)$. Jackson et $\mathrm{l}^{28}$, have also reported a low incidence of CME after cataract extraction in patients with retinitis pigmentosa. We believe that including these patients in our cohort did not alter our observations on the prevalence of CME.

We did not perform genetic analysis on all our patients, therefore we are unable to conclude whether CME is more frequent in any particular genetic subtypes. We have not analyzed any difference of visual acuity between patients with or those without CME because in Usher syndrome patients visual acuity may be affected by causes other than CME such as the formation of cataracts or intrinsic degeneration of the macular cones related to the primary genetic defect that causes the disease. Further, a substantially greater patient population would have to be analyzed to control the effect of age on visual acuity in such patients.

To conclude, we found that twenty-five per cent of our total cohort of Usher II patients had cystic-appearing macular lesions in at least one eye. Further, cysticappearing macular lesions by OCT testing were observed in over $22 \%$ of our patients with a funduscopically normal-appearing macula (four of 18). Therefore, due to the notable prevalence of CME in type II Usher patients, it would seem prudent to evaluate them for the presence of CME.

\section{Acknowledgements}

Supported by funds from the Foundation Fighting Blindness, Owings Mills Maryland, USA; the Grant Healthcare Foundation, Lake Forest, Illinois; NIH core Grant YO 1792 and an unrestricted departmental grant from Research to Prevent Blindness. 


\section{References}

1 Fishman GA, Kumar A, Joseph ME, Torok N, Anderson RJ. Usher's syndrome. Ophthalmic and neuro-otologic findings suggesting genetic heterogeneity. Arch Ophthalmol 1983; 101: 1367-1374.

2 Hope CI, Bundey S, Proops D, Fielder AR. Usher syndrome in the city of Birmingham - prevalence and clinical classification. Br J Ophthalmol 1997; 81: 46-53.

3 Rosenberg T, Haim M, Hauch AM, Parving A. The prevalence of Usher syndrome and other retinal dystrophy-hearing impairment associations. Clin Genet 1997; 51: 314-321.

4 Kremer H, van Wijk E, Märker T, Wolfrum U, Roepman R. Usher syndrome: molecular links of pathogenesis, proteins and pathways. Hum Mol Genet 2006; 15 Spec No 2: R262-R270.

5 Smith RJ, Berlin CI, Hejtmancik JF, Keats BJ, Kimberling WJ, Lewis RA et al. Clinical diagnosis of the Usher syndromes.

Usher Syndrome Consortium. Am J Med Genet 1994; 50: 32-38.

6 Tsilou ET, Rubin BI, Caruso RC, Reed GF, Pikus A, Hejtmancik JF et al. Usher syndrome clinical types I and II: could ocular symptoms and signs differentiate between the two types? Acta Ophthalmol Scand 2002; 80: 196-201.

7 Piazza L, Fishman GA, Farber M, Anderson RJ. Visual acuity loss in patients with Usher's syndrome. Arch Ophthalmol 1986; 104: 1336-1339.

8 Fishman GA, Anderson RJ, Lam BL, Derlacki DJ. Prevalence of foveal lesions in type 1 and type 2 Usher's syndrome. Arch Ophthalmol 1995; 113: 770-773.

9 Stanga PE, Downes SM, Ahuja RM, Chong NH, Antcliff R, Reck AC et al. Comparison of optical coherence tomography and fluorescein angiography in assessing macular edema in retinal dystrophies: preliminary results. Int Ophthalmol 2001; 23: 321-325.

10 Hee MR, Puliafito CA, Wong C, Duker JS, Reichel E, Schuman JS et al. Optical coherence tomography of macular holes. Ophthalmology 1995; 102: 748-756.

11 Hirakawa H, Iijima H, Gohdo T, Tsukahara S. Optical coherence tomography of cystoid macular edema associated with retinitis pigmentosa. Am J Ophthalmol 1999; 128: 185-191.

12 Apushkin MA, Fishman GA, Janowicz MJ. Monitoring cystoid macular edema by optical coherence tomography in patients with retinitis pigmentosa. Ophthalmology 2004; 111: 1899-1904.

13 Chung H, Hwang JU, Kim JG, Yoon YH. Optical coherence tomography in the diagnosis and monitoring of cystoid macular edema in patients with retinitis pigmentosa. Retina 2006; 26: 922-927.

14 Haouchine B, Massin P, Gaudric A. Foveal pseudocyst as the first step in macular hole formation: a prospective study by optical coherence tomography. Ophthalmology 2001; 108: 15-22.
15 Ghoraba H. Types of macular holes encountered during diabetic vitrectomy. Retina 2002; 22: 176-182.

16 Moldow B, Sander B, Larsen M, Engler C, Li B, Rosenberg T et al. The effect of acetazolamide on passive and active transport of fluorescein across the blood-retina barrier in retinitis pigmentosa complicated by macular oedema. Graefes Arch Clin Exp Ophthalmol 1998; 236: 881-889.

17 Giusti C, Forte R, Vingolo EM. Deflazacort treatment of cystoid macular edema in patients affected by Retinitis Pigmentosa: a pilot study. Eur Rev Med Pharmacol Sci 2002; 6: 1-8.

18 García-Arumí J, Martinez V, Sararols L, Corcostegui B. Vitreoretinal surgery for cystoid macular edema associated with retinitis pigmentosa. Ophthalmology 2003; 110: 1164-1169.

19 Fishman GA, Gilbert LD, Fiscella RG, Kimura AE, Jampol LM. Acetazolamide for treatment of chronic macular edema in retinitis pigmentosa. Arch Ophthalmol 1989; 107: $1445-1452$.

20 Newsome DA, Blacharski PA. Grid photocoagulation for macular edema in patients with retinitis pigmentosa. Am J Ophthalmol 1987; 103: 161-166.

21 Ozdemir H, Karacorlu M, Karacorlu S. Intravitreal triamcinolone acetonide for treatment of cystoid macular oedema in patients with retinitis pigmentosa. Acta Ophthalmol Scand 2005; 83: 248-251.

22 Grover S, Apushkin MA, Fishman GA. Topical dorzolamide for the treatment of cystoid macular edema in patients with retinitis pigmentosa. Am J Ophthalmol 2006; 141: 850-858.

23 Scorolli L, Morara M, Meduri A, Reggiani LB, Ferreri G, Scalinci SZ et al. Treatment of cystoid macular edema in retinitis pigmentosa with intravitreal triamcinolone. Arch Ophthalmol 2007; 125: 759-764.

24 Fishman GA, Cunha-Vaz J, Salzano T. Vitreous fluorophotometry in patients with retinitis pigmentosa. Arch Ophthalmol 1981; 99: 1202-1207.

25 Mallick KS, Zeimer RC, Fishman GA, Blair NP, Anderson RJ. Transport of fluorescein in the ocular posterior segment in retinitis pigmentosa. Arch Ophthalmol 1984; 102: 691-696.

26 Heckenlively JR, Jordan BL, Aptsiauri N. Association of antiretinal antibodies and cystoid macular edema in patients with retinitis pigmentosa. Am J Ophthalmol 1999; 127: 565-573.

27 Schwartz SB, Aleman TS, Cideciyan AV, Windsor EA, Sumaroka A, Roman AJ et al. Disease expression in Usher syndrome caused by VLGR1 gene mutation (USH2C) and comparison with USH2A phenotype. Invest Ophthalmol Vis Sci 2005; 46: 734-743.

28 Jackson H, Garway-Heath D, Rosen P, Bird AC, Tuft SJ. Outcome of cataract surgery in patients with retinitis pigmentosa. Br J Ophthalmol 2001; 85: 936-938. 